\title{
SNPP VIIRS RSB EARTH VIEW REFLECTANCE UNCERTAINTY
}

\author{
Ning Lei ${ }^{1}$, Kevin Twedt ${ }^{1}$, Jeff McIntire ${ }^{1}$, and Xiaoxiong Xiong ${ }^{2}$
}

1. Science Systems and Applications, Inc., 10210 Greenbelt Road, Suite 600, Lanham, MD 20706 USA

2. Sciences and Exploration Directorate, NASA Goddard Space Flight Center, Greenbelt, MD 20771 USA

\begin{abstract}
The Visible Infrared Imaging Radiometer Suite (VIIRS) on the Suomi National Polar-orbiting Partnership (SNPP) satellite uses its 14 reflective solar bands to passively collect solar radiant energy reflected off the Earth. The Level 1 product is the geolocated and radiometrically calibrated topof-the-atmosphere solar reflectance. The absolute radiometric uncertainty associated with this product includes contributions from the noise associated with measured detector digital counts and the radiometric calibration bias. Here, we provide a detailed algorithm for calculating the estimated standard deviation of the retrieved top-of-theatmosphere spectral solar radiation reflectance.
\end{abstract}

Index Terms - SNPP, VIIRS, radiometric, calibration, uncertainty

\section{INTRODUCTION}

One of the five remote sensing instruments onboard the Suomi National Polar-orbiting Partnership (SNPP) satellite is the Visible Infrared Imaging Radiometer Suite (VIIRS). The Level 1 data product for the reflective solar bands (RSBs) of the VIIRS, with design band wavelengths from 0.412 to $2.25 \mu \mathrm{m}$, is the geolocated and radiometrically calibrated top-of-the-atmosphere (TOA) spectral solar radiation reflectance. It is important to estimate the absolute accuracy of the retrieved reflectance to compare with the $2.0 \%$ uncertainty requirement [1]. Here, we show the algorithms for the retrieved reflectance uncertainty, measured by the estimated relative standard deviation.

\section{METHODOLOGY}

To calculate the standard deviation for the reflectance, we start with the equation [2]

$$
\rho=\frac{\pi \int L_{a p}(\lambda, t) \operatorname{RSR}(\lambda, \mathrm{t}) d \lambda \times 4 \pi R^{2}(t)}{\cos \left(\theta_{\text {earth }- \text { sun }}(t)\right) \int \Phi_{\text {sun }}(\lambda, t) \operatorname{RSR}(\lambda, \mathrm{t}) d \lambda},
$$

where $L_{a p}(\lambda, t)$ is the retrieved scene TOA spectral radiance, RSR is the relative spectral response, $\theta_{\text {earth - sun }}$ is the angle between the solar vector and the scene surface normal, $\Phi_{\text {sun }}$ is the solar spectral power, and $R$ is the distance between the VIIRS and the Sun. The RSR is averaged over all detectors in a band. The retrieved scene spectral radiance $L_{a p}(\lambda, t)$ is calculated by

$$
\frac{\int L_{a p}(\lambda, t) \operatorname{RSR}(\lambda, \mathrm{t}) d \lambda}{\int \operatorname{RSR}(\lambda, \mathrm{t}) d \lambda}=\frac{F \times\left(c_{0}+c_{1} d n_{E V}+c_{2} d n_{E V}^{2}\right)}{\operatorname{RVS}\left(\theta_{\mathrm{EV}}\right)} .
$$

In Eq. (2), RVS is the reflectivity of the half-angle-mirror (HAM) relative to the value when the rotating telescope assembly (RTA) observes the onboard solar diffuser (SD), $\theta_{\mathrm{EV}}$ is the angle between the HAM surface normal and the incident light, $F$ is a correction factor so that the right hand side of Eq. (2) gives the correct scene spectral radiance, $\left(c_{0}, c_{1}, c_{2}\right)$ are determined prelaunch, and $d n_{E V}$ is the background subtracted detector digital count for the scene.

The $F$ in Eq. (2) is calibrated through observations of the fully solar illuminated SD $[2,3]$

$$
\begin{aligned}
& F\left(t^{\prime}\right)= \\
& {\left[\begin{array}{l}
\cos \left(\theta_{S D-\text { sun }}\left(t^{\prime}\right)\right) \tau\left(\vec{\phi}_{\text {sun }}\left(t^{\prime}\right)\right) \times \\
\operatorname{BRDF} \operatorname{RTA}_{\operatorname{RT}}\left(\lambda, \vec{\phi}_{\text {sun }}, t=0\right) \mathrm{H}_{\mathrm{RTA}}\left(\lambda, \vec{\phi}_{\text {sun }}, t^{\prime}\right) \times \\
\Phi \Phi_{\text {sun }}\left(\lambda, t^{\prime}\right) \mathrm{RSR}\left(\lambda, t^{\prime}\right) d \lambda
\end{array}\right]} \\
& 4 \pi R^{2}\left(t^{\prime}\right) c_{1}\left[c_{0} / c_{1}+d n_{S D}+\left(c_{2} / c_{1}\right) \times d n_{S D}^{2}\right] \mathrm{RSR}(\lambda) d \lambda
\end{aligned}
$$

In Eq. (3), $\theta_{S D-s u n}$ is the angle between the SD surface normal and the solar vector, $\tau$ is SD screen transmittance, $\vec{\phi}_{\text {sun }}$ is the solar angle, BRDF ${ }_{\text {RTA }}$ is the SD bidirectional reflectance distribution function at the RTA-SD view direction at launch, $\mathrm{H}_{\mathrm{RTA}}$ is the relative change in the BRDF RTA since launch, and $d n_{S D}$ is the background subtracted digital counts when the sensor observes the fully sunlit SD. The $t$ ' in Eq. (3) and the $t$ in Eq. (2) should be close enough so that the $F$ is assumed to remain constant over the time interval.

Considering the small separation between $t$ and $t^{\prime}$, $\Phi_{\text {sun }}(\lambda, t)$ and $\Phi_{\text {sun }}\left(\lambda, t^{\prime}\right)$ are essentially the same and for the purpose of calculating the variance of the retrieved reflectance, we need to consider only the wavelength integral over the RSR central peak. We combine Eqs. (1-3) to obtain the spectral reflectance as 


$$
\begin{aligned}
& \rho=\frac{\pi}{\operatorname{RVS}\left(\theta_{\mathrm{EV}}\right)} \times \frac{\cos \theta_{S D-\operatorname{sun}}\left(t^{\prime}\right) \tau\left(\vec{\phi}_{\text {sun }}\left(t^{\prime}\right)\right)}{\cos \left(\theta_{\text {earth }}-\operatorname{sun}(t)\right)} \times \\
& \frac{\frac{c_{0}}{c_{1}}+d n_{E V}+\frac{c_{2}}{c_{1}} d n_{E V}^{2}}{\frac{c_{0}}{c_{1}}+d n_{S D}+\frac{c_{2}}{c_{1}} d n_{S D}^{2}} \times \frac{c_{1}(t)}{c_{1}\left(t^{\prime}\right)} \times \frac{R^{2}(t)}{R^{2}\left(t^{\prime}\right)} \times \\
& \mathrm{BRDF}_{\mathrm{RTA}}\left(\lambda_{B}, \vec{\phi}_{\text {sun }}, t=0\right) \times H_{\mathrm{RTA}}\left(\lambda_{B}, \vec{\phi}_{\text {sun }}, t\right)
\end{aligned}
$$

We use the prelaunch error estimate for the RVS, which has a standard deviation of less than $0.1 \%$ for the RSBs [4]. The $c_{1}$ ratio, affected mainly by the detector focal plane temperature variation, differs from one by less than $0.1 \%$ because the focal plane temperature varies by less than 0.5 degrees over the daylight time of an orbit and the temperature's impact on $c_{1}$ is less than $1 \%$ for every 10 degrees of the temperature change [5]. Additionally, the VIIRS-Sun distance is accurately known, with a relative error less than $10^{-8}$. The noise in $d n_{\mathrm{SD}}$ is small since on a per scan basis it is the average over 48 RTA angular positions and further effectively averaged over many scans on a single satellite orbit. Furthermore, the co-variances are either zero or negligibly small. Hence, we write the relative variance of the TOA reflectance as

$$
\begin{aligned}
& \frac{\operatorname{var}(\rho)}{\rho^{2}}=\frac{\operatorname{var}\left(\cos \theta_{S D-\text { sun }}\right)}{\cos ^{2} \theta_{S D-\text { sun }}}+\frac{\operatorname{var}\left(\cos \theta_{\text {earth }- \text { sun }}\right)}{\cos ^{2} \theta_{\text {earth }}-\text { sun }}+ \\
& \frac{\operatorname{var}\left(\frac{c_{0}}{c_{1}}+d n_{E V}+\frac{c_{2}}{c_{1}} d n_{E V}^{2}\right)}{\left(\frac{c_{0}}{c_{1}}+d n_{E V}+\frac{c_{2}}{c_{1}} d n_{E V}^{2}\right)^{2}}+\frac{\operatorname{var}\left(\mathrm{H}_{\mathrm{RTA}}\left(\lambda_{B}, \vec{\phi}_{\text {sun }}, t\right)\right)}{\left(\mathrm{H}_{\mathrm{RTA}}\left(\lambda_{B}, \vec{\phi}_{\text {sun }}, t\right)\right)^{2}}+. \\
& \frac{\operatorname{var}\left(\operatorname{BRDF} \text { RTA }_{B}\left(\lambda_{B}, \vec{\phi}_{\text {sun }}, t=0\right) \tau\left(\vec{\phi}_{\text {sun }}\right)\right)}{(\operatorname{BRDF}}
\end{aligned}
$$

We calculate each term on the right hand side of Eq. (5).

The first term is $\tan ^{2}\left(\theta_{S D-s u n}\right) \operatorname{var}\left(\theta_{S D-s u n}\right)$. During the calibration data collection process, $\theta_{S D-\text { sun }}$ varies from 50 to 59 degrees. $\operatorname{var}\left(\theta_{S D-s u n}\right)$ has contributions from the errors in the solar vector direction and the SD surface normal vector. The solar vector direction has a standard deviation of about 0.001 prior to February 23, 2012 and about 0.00008 afterward [6]. We estimate that the standard deviation of the SD surface normal vector error is much less than 0.001 and thus we ignore this error. As a result, $\operatorname{var}\left(\theta_{S D-s u n}\right)$ is only marginally significant prior to February 23, 2012 and negligibly small afterward.

The second term is $\tan ^{2}\left(\theta_{\text {earth }- \text { sun }}\right)$ var $\left(\theta_{\text {earth }- \text { sun }}\right)$. Similar to $\theta_{S D-s u n}$, the error in $\theta_{\text {earth - sun }}$ is primarily caused by the solar vector directional error and thus var $\left(\theta_{\text {earth }}-\right.$ sun $)$ is about $0.001^{2}$ prior to February 23, 2012 and about $0.00008^{2}$ afterward. Hence, the second term does not contribute much to the variance of the retrieved reflectance until $\theta_{\text {earth }- \text { sun }}$ is close to $\pm 90^{\circ}$. For example, for the second term to be larger than $0.01^{2},\left|\theta_{\text {earth }- \text { sun }}\right|$ needs to be larger than $84.3^{\circ}$.

The third term is dominated by the linear term in $d n_{E V}$ and thus is simplified to $\operatorname{var}\left(d n_{E V}\right) / d n_{E V}^{2} \cdot d n_{E V}$ is $D N_{E V}+0.375-D N_{S V, a v g}$ with $D N_{E V}$ a 12-bit integer and $D N_{S V}$, avg is typically the average of $48 \mathrm{SV}$ digital numbers in the same scan. The 0.375 is added to the 12-bit $D N_{E V}$ to remove the bias due to the truncation. $D N_{S V}$ at each sample is a 14-bit number with 2 bits for the fraction. The truncation of $D N_{E V}$ to 12-bit adds roughly 1/12 to the original analog signal variance. Mathematically, we write

$\operatorname{var}\left(d n_{E V}\right)=\operatorname{var}\left(D N_{E V}\right)+\operatorname{var}\left(D N_{S V, a v g}\right)$
$=\operatorname{var}\left(D N_{E V}(\operatorname{analog})\right)+1 / 12+\operatorname{var}\left(D N_{S V, a v g}\right)$

We calculate the original signal variance $\operatorname{var}\left(D N_{E V}(\right.$ analog $\left.)\right)$ from the variance of a single sample $d n_{S D}$ as

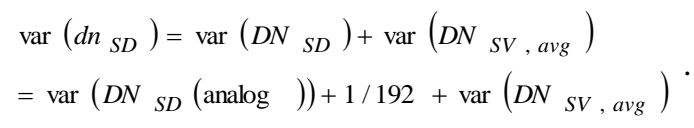

In Eq. (7), 1/192 is the additional variance due to the digitization of $D N_{S D}$, a 14-bit number with 2 bits for the fraction. Combining Eqs. (6) and (7) by taking $\operatorname{var}\left(D N_{E V}(\operatorname{analog})\right)=\operatorname{var}\left(D N_{S D}(\operatorname{analog})\right) \quad$ when $D N_{E V}+0.375=D N_{S D}$ (roughly when $d n_{S D}$ matches $d n_{E V} \quad$ in strength), we retrieve $\operatorname{var}\left(d n_{E V}\right)$ by $\operatorname{var}\left(d n_{E V}\right)=\operatorname{var}\left(d n_{S D}\right)+1 / 12-1 / 192$.

We would like to use a simple function of $d n_{E V}$ to calculate $\operatorname{var}\left(d n_{E V}\right)$. Considering the white noise which includes the thermal and digitization noises and the Poisson noise as the main contributions to the noise in $d n_{E V}$, we use the functional form of [7] $\operatorname{var}\left(d n_{E V}\right)=k_{0}+k_{1} \times d n_{E V}$, where $k_{0}$ and $k_{1}$ are associated with the white and the Poisson noises, respectively, and are approximately $d n_{E V}$ independent. This linear functional dependence is demonstrated by Fig. 1 that shows the variance vs. $d n_{\mathrm{SD}}$ for 
detector 8 of band M6 at orbit 25986. var $\left(d n_{E V}\right) / d n_{E V}^{2}$ at $\mathrm{L}_{\text {typ }}$ at this writing are larger than $201^{-2}$ for the VISNIR bands at the high-gain stage for dual-gain bands, and $137^{-2}$ for the SWIR bands, except the M11 band for which $\operatorname{var}\left(d n_{E V}\right) / d n_{E V}^{2}$ is about $21^{-2}$.

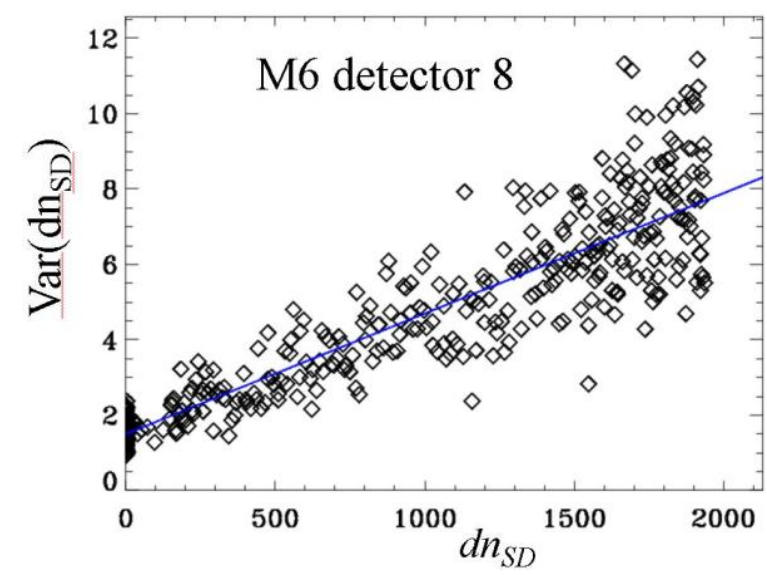

Fig. 1. Variance vs. $d n_{\mathrm{SD}}$ for detector 8 of band M6 on satellite orbit 25986.

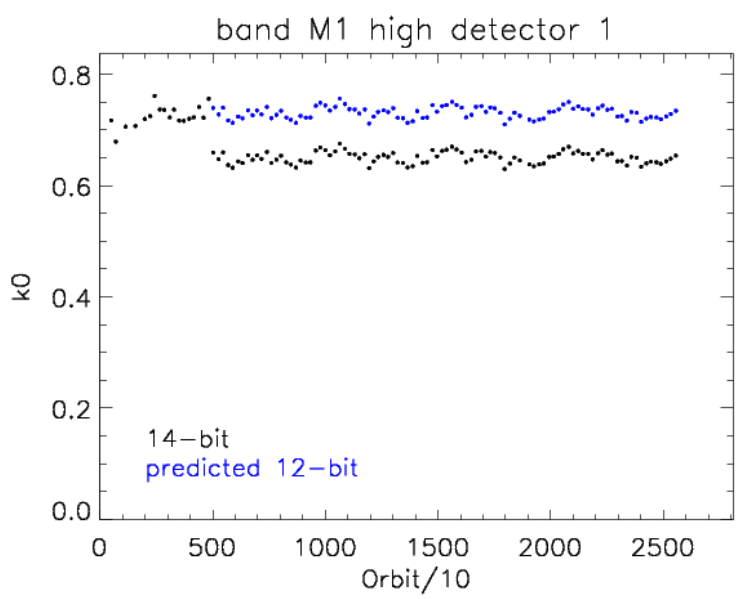

Fig. 2. $k_{0}$ versus satellite orbit numbers for the M1 band detector 1 at the high-gain stage. The blue dots are for the $k_{0}$ obtained from a least-squares fit with the retrieved $\operatorname{var}\left(d n_{E V}\right)$. The black dots are for $k_{0}$ obtained from a least-squares fit to the SD data without the digitization correction. Near the mission start, the black dots have higher values since prior to orbit around 5010 $d n_{S D}$ was a 12-bit integer and afterward has become a 14-bit number with 2 bits for the fraction.

We determine $k_{0}$ and $k_{1}$ by performing a least-squares fit to the SD data over a wide range of signal levels. In Fig. 2, we show an example of $k_{0}$ versus satellite orbit number. The curve shows that $k_{0}$ may be described by a linear function of time, $k_{0}=a_{0}+a_{1} \times$ orbit . Similarly, we may describe $k_{1}$ by a linear function of time, $k_{1}=b_{0}+b_{1} \times$ orbit , as suggested by Fig. 3 . We tabulate $a_{0}, a_{1}, b_{0}$, and $b_{1}$ for each detector, gain, and band; $k_{0}$ and $k_{1}$ are not HAM dependent because they are independent of $d n_{E V}$.

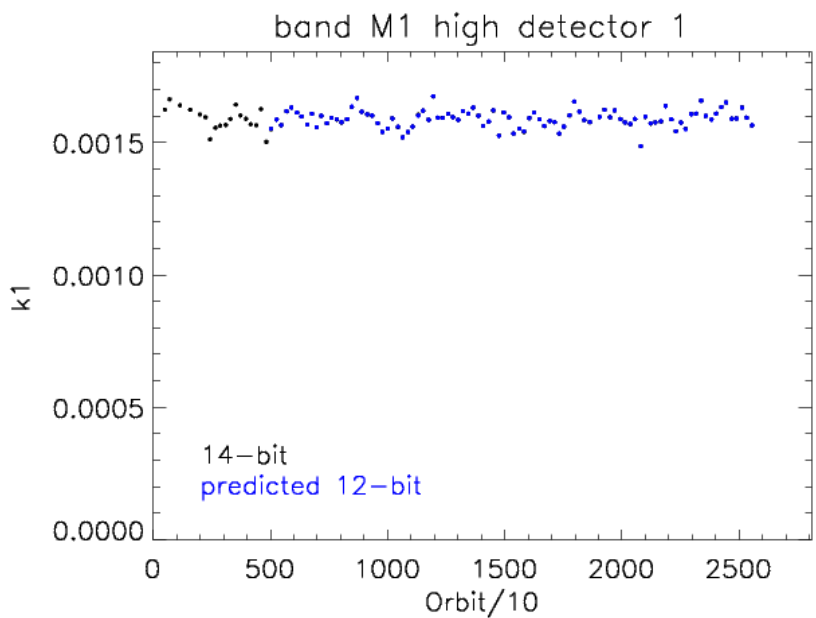

Fig. 3. $k_{1}$ versus satellite orbit numbers for the M1 band detector 1 at the high-gain stage. The blue dots are for the $k_{1}$ obtained from a least-squares fit with the retrieved $\operatorname{var}\left(d n_{E V}\right)$ and the black dots are $k_{1}$ without the digitization correction. Since the digitization correction is independent of signal level, it has no impact on $k_{1}$.

The fourth term on the right hand side of Eq. (5) is related to the uncertainty of $H_{\text {RTA }}$. This uncertainty is influenced by the uncertainty of $H_{\text {SDSM }}$ and the $F$ calculated with lunar observation data, as well as the SD positional dependence of $H_{\text {RTA }}$. The $F$ from the lunar data tells us how much $H_{\text {RTA }}$ differs from $H_{\text {SDSM }}$. The multi-year lunar $F$ relative to its value at a particular time has an accuracy of about $0.1 \%$. We approximate that the accuracy of $H_{\text {SDSM }}$ is less than $0.1 \%$, except at the M1 band central wavelength where the accuracy is about $0.001^{2}+0.0005^{2} / \mathrm{H}_{\mathrm{RTA}}{ }^{2}$ [9]. Note that the positional dependence of $H_{\mathrm{RTA}}$ has not been estimated. As a result, we write the relative variance of $H_{\mathrm{RTA}}$ as $2 \times 0.001^{2} / H_{\mathrm{RTA}}^{2}+0.0005^{2}$ for the M1 band and $0.001^{2} / H_{\mathrm{RTA}}^{2}$ for the rest of the RSBs.

The fifth term on the right hand side of Eq. (5) is dominated by the errors in the prelaunch $\mathrm{BRDF}_{\mathrm{RTA}}$ [8]. For each angular data point, the prelaunch $\tau$ has a relative uncertainty of $0.24 \%$. The relative value of $\operatorname{BRDF}_{\mathrm{RTA}}\left(\lambda_{B}, \vec{\phi}_{\text {sun }}, t=0\right) \tau$ is determined by on-orbit yaw maneuver data (further improvements with regular on-orbit data have been made but are not used in this study) and the relative $\operatorname{BRDF} \operatorname{RTA}_{B}\left(\lambda_{B}, \vec{\phi}_{\text {sun }}, t=0\right) \tau$ is scaled to match the prelaunch BRDF ${ }_{\text {RTA }} \tau$. Consequently, we effectively 
average the prelaunch $\tau$ over many angular data points and the uncertainty of this average should be much smaller than $0.24 \%$ and is ignored. Bias in the prelaunch $\tau$ is not estimated here. The prelaunch $\mathrm{BRDF}_{\mathrm{RTA}}$ has a relative variance of $0.011^{2}+0.005^{2}$ for the VISNIR bands and $0.013^{2}+0.005^{2}$ for the SWIR bands [8]. We added $0.005^{2}$ to the originally given prelaunch $\mathrm{BRDF}_{\mathrm{RTA}}$ variance to account for the additional uncertainty associated with the possible change in the BRDF between prelaunch BRDF RTA $_{1}$ measurement time and the satellite launch time.

Hence, when the angle between the sunlight and the Earth surface normal is not close to $\pm 90^{\circ}$, for non-aggregated Earth views, the reflectance uncertainties for the RSBs at $\mathrm{L}_{\text {typ }}$, as measured by $s t d(\rho) / \rho$, are dominated by $d n_{\mathrm{EV}}$ noise and the prelaunch BRDF uncertainties. At $\mathrm{L}_{\text {typ }}$, at the current time, the uncertainty is less than $1.3 \%$ for the VISNIR bands, and $1.6 \%$ for the SWIR bands, except for the M11 band which has an uncertainty of about $4.9 \%$.

\section{AGGREGATION IMPACT}

Since VIIRS aggregates samples together for some scan angles, we need to consider the impact of the aggregation on the relative variance of the retrieved TOA reflectance. The aggregation for the dual-gain bands happens after the radiometric calibration for each sample is performed and thus the average is performed on the retrieved scene reflectance. As a result, the variance contribution from $d n_{E V}$ is given by

$$
\operatorname{var}\left(d n_{E V} ; \mathrm{DG}\right)=\sum_{i=1}^{N_{\text {agg }}}\left[k_{0}\left(G_{i}\right)+k_{1}\left(G_{i}\right) d n_{E V}(i)\right] / N_{a g g}^{2},
$$

where DG indicates a dual-gain band, $N_{\text {agg }}$ indicates the number of per sample $d n_{E V}$ to be averaged, and $G_{i}$ indicates the detector gain stage.

For the single-gain bands, the aggregation happens before the digital number is truncated to a 12-bit integer and we have

$$
\begin{aligned}
\operatorname{var}\left(d n_{E V} ; S G\right)= & {\left[\sum_{i=1}^{N_{a g g}}\left(k_{0}+k_{1} d n_{E V, i}\right)\right] / N_{a g g}^{2}+} \\
& {\left[1 / 12+\operatorname{var}\left(D N_{S V, a v g}\right)\right] \times\left(1-1 / N_{a g g}\right) }
\end{aligned}
$$

\section{SUMMARY}

We showed the mathematical expressions to calculate the relative variance of the retrieved TOA solar radiation spectral reflectance for the SNPP VIIRS RSBs. The variance is dominated by the variance of the prelaunch SD BRDF at the RTA direction when the scene signal level is high. The white and Poisson noises contribute significantly to the relative variance when the signal level is low. Possible biases in the SD positional dependence of the BRDF degradation factor and the prelaunch SD screen transmittance and BRDF are ignored. The uncertainty can be easily calculated with a few time-independent parameters and the measured background subtracted detector digital count. We determined the values of the time-independent parameters from observations of the sunlit SD. Additionally, we derived mathematical expressions to calculate the reflectance variance when detector samples are aggregated. The formulas allow us to implement the uncertainty as part of the Level one product on a per (pixel, detector) basis. When the angle between the sunlight and the Earth surface normal is not close to $\pm 90^{\circ}$, for non-aggregated Earth views, the reflectance uncertainties for the RSBs at the respective typical scene spectral radiances are less than $1.3 \%$ for the VISNIR bands and $1.6 \%$ for the SWIR bands, except for the M11 band which has an uncertainty of about $4.9 \%$.

\section{REFERENCES}

[1] R. M. Durham, "Sensor specification for the visible infrared imager radiometer suite," Raytheon Systems Company, Goleta, CA, USA, Tech. Rep. PRT PS 154640-101A, 2002.

[2] Joint Polar Satellite System (JPSS) VIIRS Radiometric Calibration Algorithm Theoretical Basis Document (ATBD); NASA Goddard Space Flight Center: Greenbelt, MD, USA, 2013.

[3] N. Lei, Z. Wang, and X. Xiong, "On-orbit Radiometric Calibration of Suomi NPP VIIRS Reflective Solar Bands through Observations of a Sunlit Solar Diffuser Panel", IEEE Trans. Geosci. Remote Sens., vol. 53, pp. 5983-5990, 2015, DOI 10.1109/TGRS.2015.2430814

[4] A. Wu, X. Xiong, and C. Cao, "Tracking on-orbit stability of the response versus scan angle for the S-NPP VIIRS reflective solar bands", Proc. SPIE, vol. 9972, Art ID:9972-1C, 2016, doi: 10.1117/12.2238106.

[5] N. Lei and X. Xiong, "Functional form of the radiometric equation for the SNPP VIIRS reflective solar bands", Proc. SPIE, vol. 9972, Art ID. 9972-19, 2016; doi:10.1117/12.2236950.

[6] R. Wolfe, G. Lin, M. Nishihama, K. Tewari, J. Tilton, and A, Issacman, "Suomi NPP VIIRS prelaunch and on-orbit geometric calibration and characterization", JGR: Atmos., vol 118, 2013.

[7] N. Lei, X. Xiong, and B. Guenther, "Modeling the Detector Radiometric Gains of the Suomi NPP VIIRS Reflective Solar Bands", IEEE Trans. Geosci. Remote Sens., vol. 53, pp. 15651573, 2015, DOI: 10.1109/TGRS.2014.2345481.

[8] L. Lessel and S. McClain, "Low Uncertainty Measurements of Bidirectional Reflectance Factor on the NPOESS/VIIRS Solar Diffuser", Proc. SPIE, vol. 6677, Art ID 6677-1O, 2007.

[9] N. Lei and X. Xiong, "Estimation of the Accuracy of the SNPP VIIRS SD BRDF Degradation Factor Determined by the Solar Diffuser Stability Monitor", Proc. SPIE, vol. 9607, Art ID. $9607-$ $1 \mathrm{~V}, 2015$. 